\title{
Direct constructions of hyperplanes of dual polar spaces arising from embeddings
}

\author{
Bart De Bruyn* \\ Ghent University, Department of Pure Mathematics and Computer Algebra, \\ Krijgslaan 281 (S22), B-9000 Gent, Belgium, E-mail: bdb@cage.ugent.be
}

\begin{abstract}
Let $e$ be one of the following full projective embeddings of a finite dual polar space $\Delta$ of rank $n \geq 2$ : (i) the Grassmann-embedding of the symplectic dual polar space $\Delta \cong D W(2 n-1, q)$; (ii) the Grassmannembedding of the Hermitian dual polar space $\Delta \cong D H\left(2 n-1, q^{2}\right)$; (iii) the spin-embedding of the orthogonal dual polar space $\Delta \cong$ $D Q(2 n, q)$; (iv) the spin-embedding of the orthogonal dual polar space $\Delta \cong D Q^{-}(2 n+1, q)$. Let $\mathcal{H}_{e}$ denote the set of all hyperplanes of $\Delta$ arising from the embedding $e$. We give a method for constructing the hyperplanes of $\mathcal{H}_{e}$ without implementing the embedding $e$ and discuss (possible) applications of the given construction.
\end{abstract}

Keywords: dual polar space, hyperplane, Grassmann-embedding, spin-embedding MSC2000: 51A45, 51A50, 51E20

\section{Introduction}

\subsection{Basic definitions}

Let $\Pi$ be a non-degenerate polar space of rank $n \geq 2$. With $\Pi$, there is associated a point-line geometry $\Delta$ whose points are the maximal singular subspaces of $\Pi$, whose lines are the next-to-maximal singular subspaces of $\Pi$ and whose incidence relation is reverse containment. We call $\Delta$ a dual polar space (Cameron [4]). The dual polar space $\Delta$ is a near polygon (Shult and Yanushka [31]; De Bruyn [13]) which means that for every point $p$ and every

${ }^{*}$ Postdoctoral Fellow of the Research Foundation - Flanders (Belgium) 
line $L$, there exists a unique point $\pi_{L}(p)$ on $L$ nearest to $p$. Here, distances $\mathrm{d}(\cdot, \cdot)$ are measured in the point or collinearity graph of $\Delta$. For every point $x$ of $\Delta$, for every non-empty subset $X$ of the point-set $P$ of $\Delta$ and for every $i \in$ $\mathbb{N}$, we define $\Delta_{i}(x):=\{y \in P \mid \mathrm{d}(x, y)=i\}, \Delta_{\leq i}(x):=\{y \in P \mid \mathrm{d}(x, y) \leq i\}$, $x^{\perp}:=\Delta_{\leq 1}(x), \mathrm{d}(x, X)=\min \{\mathrm{d}(x, y) \mid y \in X\}, \Delta_{i}(X)=\{y \in P \mid \mathrm{d}(y, X)=$ $i\}$. If $X_{1}$ and $X_{2}$ are two non-empty sets of points of $\Delta$, then we define $\mathrm{d}\left(X_{1}, X_{2}\right):=\min \left\{\mathrm{d}\left(x_{1}, x_{2}\right) \mid x_{1} \in X_{1}\right.$ and $\left.x_{2} \in X_{2}\right\}$. One of the following two cases occurs for two lines $K_{1}$ and $K_{2}$ of $\Delta$ : (1) there exist unique points $x_{1} \in K_{1}$ and $x_{2} \in K_{2}$ such that $\mathrm{d}\left(y_{1}, y_{2}\right)=\mathrm{d}\left(y_{1}, x_{1}\right)+\mathrm{d}\left(x_{1}, x_{2}\right)+\mathrm{d}\left(x_{2}, y_{2}\right)$ for every $\left(y_{1}, y_{2}\right) \in K_{1} \times K_{2} ;(2)$ for every $\left(y_{1}, y_{2}\right) \in K_{1} \times K_{2}, \mathrm{~d}\left(y_{1}, K_{2}\right)=$ $\mathrm{d}\left(y_{2}, K_{1}\right)=\mathrm{d}\left(K_{1}, K_{2}\right)$. If case (2) occurs, then the lines $K_{1}$ and $K_{2}$ are called parallel (notation $K_{1} \| K_{2}$ ).

There exists a bijective correspondence between the non-empty convex subspaces of a dual polar space $\Delta$ of rank $n \geq 2$ and the possibly empty singular subspaces of its associated polar space $\Pi$. If $\alpha$ is a singular subspace of $\Pi$, then the set of all maximal singular subspaces containing $\alpha$ is a convex subspace of $\Delta$. Conversely, every convex subspace of $\Delta$ is obtained in this way. The maximal distance between two points of a convex subspace $A$ is called the diameter of $A$. The convex subspaces of diameter 2, 3, respectively $n-1$, are called the quads, hexes, respectively maxes, of $\Delta$. The convex subspaces through a given point $x$ of $\Delta$ determine a projective space of dimension $n-1$ which we will denote by $\operatorname{Res}_{\Delta}(x)$. If $x$ and $y$ are two points of $\Delta$, then $\langle x, y\rangle$ denotes the smallest convex subspace containing $x$ and $y$, i.e. $\langle x, y\rangle$ is the intersection of all convex subspaces containing $x$ and $y$. More generally, if $*_{1}, \ldots, *_{k}$ are objects of $\Delta$ like points or sets of points, then $\left\langle *_{1}, *_{2}, \ldots, *_{k}\right\rangle$ denotes the smallest convex subspace of $\Delta$ containing $*_{1}, *_{2}, \ldots, *_{k}$. If $x$ is a point and $A$ is a non-empty convex subspace of $\Delta$, then $A$ contains a unique point $\pi_{A}(x)$ nearest to $x$ and $\mathrm{d}(x, y)=$ $\mathrm{d}\left(x, \pi_{A}(x)\right)+\mathrm{d}\left(\pi_{A}(x), y\right)$ for every point $y$ of $A$. We call $\pi_{A}(x)$ the projection of $x$ on $A$.

A hyperplane of a point-line geometry $\mathcal{S}$ is a proper subspace of $\mathcal{S}$ meeting each line of $\mathcal{S}$. Suppose now that $\Delta$ is a dual polar space. If $x$ is a point of a hyperplane $H$ of $\Delta$, then $\Lambda_{H}(x)$ denotes the set of all lines of $\Delta$ through $x$ contained in $H$. Notice that we can regard $\Lambda_{H}(x)$ as a set of points of the projective space $\operatorname{Res}_{\Delta}(x)$. A point $x$ of a hyperplane $H$ of $\Delta$ is called deep if $x^{\perp} \subseteq H$, or equivalently, if $\Lambda_{H}(x)$ coincides with the whole point-set of $\operatorname{Res}_{\Delta}(x)$. Since $\Delta$ is a near polygon, the set of points of $\Delta$ at non-maximal distance from a given point $x$ is a hyperplane of $\Delta$. We call this hyperplane the singular hyperplane of $\Delta$ with deepest point $x$. By Shult [30, Lemma 6.1], every hyperplane of a thick dual polar space $\Delta$ is a maximal subspace of $\Delta$, or equivalently, the complement of a hyperplane of $\Delta$ is connected. If $\Delta$ is a 
thick dual polar space and if $Q$ is a quad of $\Delta$, then either $Q \subseteq H$ or $Q \cap H$ is a hyperplane of $Q$. By Payne and Thas [26], one of the following cases then occurs:

(i) $Q \subseteq H$,

(ii) $Q \cap H=x^{\perp} \cap Q$ for some point $x$ of $Q$,

(iii) $Q \cap H$ is a proper subquadrangle of $Q$, or

(iv) $Q \cap H$ is an ovoid of $Q$, i.e. a set of points of $Q$ meeting each line of $Q$ in a unique point.

If case (i), (ii), (iii), respectively (iv), occurs, then $Q$ is called deep, singular, subquadrangular, respectively ovoidal, with respect to $H$. If every nondeep quad is singular with respect to $H$, then $H$ is called locally singular.

A full (projective) embedding of a dual polar space $\Delta$ is an injective mapping $e$ from the point-set $P$ of $\Delta$ to the point-set of a projective space $\Sigma$ satisfying (i) $\langle e(P)\rangle_{\Sigma}=\Sigma$; (ii) $e(L):=\{e(x) \mid x \in L\}$ is a line of $\Sigma$ for every line $L$ of $\Delta$. For every hyperplane $\alpha$ of $\Sigma$, the set $e^{-1}(e(P) \cap \alpha)$ is a hyperplane of $\Delta$. We say that the hyperplane $e^{-1}(e(P) \cap \alpha)$ arises from the embedding $e$. If $H$ is a hyperplane of a thick dual polar space $\Delta$ arising from an embedding $e$, then since $H$ is a maximal subspace of $\Delta,\langle e(H)\rangle_{\Sigma}$ is a hyperplane of $\Sigma$ and $\langle e(H)\rangle_{\Sigma} \cap e(P)=e(H)$.

In this paper we will meet four classes of dual polar spaces and embeddings.

(I) Let $\Pi=W(2 n-1, q)$ be the polar space of the subspaces of $\mathrm{PG}(2 n-$ $1, q), n \geq 2$, which are totally isotropic with respect to a given symplectic polarity of $\mathrm{PG}(2 n-1, q)$. Let $\Delta=D W(2 n-1, q)$ denote the associated symplectic dual polar space. It is well-known that $D W(2 n-1, q)$ has a full embedding into the projective space $\operatorname{PG}\left(\left(\begin{array}{c}2 n \\ n\end{array}\right)-\left(\begin{array}{c}2 n \\ n-2\end{array}\right)-1, q\right)$, see e.g. Cooperstein [11] or De Bruyn [15]. This embedding is called the Grassmannembedding of $D W(2 n-1, q)$.

(II) Let $\Pi=H\left(2 n-1, q^{2}\right)$ be the polar space of the subspaces of PG(2n$\left.1, q^{2}\right), n \geq 2$, which are totally isotropic with respect to a given Hermitian polarity of $\mathrm{PG}\left(2 n-1, q^{2}\right)$. Let $\Delta=D H\left(2 n-1, q^{2}\right)$ denote the associated Hermitian dual polar space. The dual polar space $D H\left(2 n-1, q^{2}\right)$ has a nice full embedding into $P G\left(\left(\begin{array}{c}2 n \\ n\end{array}\right)-1, q\right)$, see Cooperstein [10] and De Bruyn [16]. This embedding is called the Grassmann-embedding of $D H\left(2 n-1, q^{2}\right)$.

(III) Let $\Pi=Q(2 n, q)$ denote the polar space of the subspaces of $\mathrm{PG}(2 n, q)$, $n \geq 2$, which lie on a given nonsingular parabolic quadric $Q(2 n, q)$ of $\mathrm{PG}(2 n, q)$. Let $\Delta=D Q(2 n, q)$ denote the associated orthogonal dual polar space. It is well-known that $D Q(2 n, q) \cong D W(2 n-1, q)$ if and only if $q$ is even. The dual polar space $D Q(2 n, q)$ has a nice full embedding into $\mathrm{PG}\left(2^{n}-1, q\right)$, see Chevalley [9] or Buekenhout \& Cameron [3]. This embedding is called the spin-embedding of $D Q(2 n, q)$. 
(IV) Let $\Pi=Q^{-}(2 n+1, q)$ denote the polar space of the subspaces of $\mathrm{PG}(2 n+1, q), n \geq 2$, which lie on a given nonsingular elliptic quadric of $\mathrm{PG}(2 n+1, q)$. Let $\Delta=D Q^{-}(2 n+1, q)$ denote the associated orthogonal dual polar space. The dual polar space $D Q^{-}(2 n+1, q)$ has a nice full embedding into $\mathrm{PG}\left(2^{n}-1, q^{2}\right)$, see Cooperstein and Shult [12]. This embedding is called the spin-embedding of $D Q^{-}(2 n+1, q)$.

\subsection{The Main Theorem}

Suppose one of the following cases occurs:

(I) $e: \Delta \rightarrow \Sigma$ is the Grassmann-embedding of the symplectic dual polar space $\Delta \cong D W(2 n-1, q), n \geq 2$, into $\Sigma \cong \operatorname{PG}\left(\left(\begin{array}{c}2 n \\ n\end{array}\right)-\left(\begin{array}{c}2 n \\ n-2\end{array}\right)-1, q\right)$;

(II) $e: \Delta \rightarrow \Sigma$ is the Grassmann-embedding of the Hermitian dual polar space $\Delta \cong D H\left(2 n-1, q^{2}\right), n \geq 2$, into $\Sigma \cong \operatorname{PG}\left(\left(\begin{array}{c}2 n \\ n\end{array}\right)-1, q\right)$;

(III) $e: \Delta \rightarrow \Sigma$ is the spin-embedding of the orthogonal dual polar space $\Delta \cong D Q(2 n, q), n \geq 2$, into $\Sigma \cong \mathrm{PG}\left(2^{n}-1, q\right)$;

(IV) $e: \Delta \rightarrow \Sigma$ is the spin-embedding of the orthogonal dual polar space $\Delta \cong D Q^{-}(2 n+1, q), n \geq 2$, into $\Sigma \cong \mathrm{PG}\left(2^{n}-1, q^{2}\right)$.

In each of the considered cases, let $P$ denote the point-set of $\Delta$. Let $\mathcal{H}_{e}$ denote the set of all hyperplanes of $\Delta$ arising from $e$ and let $\mathcal{H}_{e}^{\prime}$ be the following set of hyperplanes of $\Delta$.

In case (I), a hyperplane $H$ of $\Delta$ belongs to $\mathcal{H}_{e}^{\prime}$ if for every quad $Q$ of $\Delta$ which is ovoidal with respect to $H, Q \cap H$ is a classical ovoid of $Q$, i.e. an elliptic quadric $Q^{-}(3, q)$ on $Q \cong Q(4, q)$.

In case (II), every hyperplane of $\Delta$ belongs to $\mathcal{H}_{e}^{\prime}$.

In case (III), the hyperplanes of $\mathcal{H}_{e}^{\prime}$ are precisely the locally singular hyperplanes of $\Delta$.

In case (IV), a hyperplane $H$ of $\Delta$ belongs to $\mathcal{H}_{e}^{\prime}$ if for every quad $Q$ of $\Delta$ which is ovoidal with respect to $H, Q \cap H$ is a classical ovoid of $Q$, i.e. a unital $H\left(2, q^{2}\right)$ on $Q \cong H\left(3, q^{2}\right)$.

In all four cases, we have $\mathcal{H}_{e} \subseteq \mathcal{H}_{e}^{\prime}$. In cases (III) and (IV), $\mathcal{H}_{e}=\mathcal{H}_{e}^{\prime}$ by De Bruyn [14]. In case (II), $\mathcal{H}_{e}=\mathcal{H}_{e}^{\prime}$ if $q>2$ and $\mathcal{H}_{e} \neq \mathcal{H}_{e}^{\prime}$ if $q=2$, see Cardinali, De Bruyn and Pasini [8, Corollary 1.6]. In case (I), $\mathcal{H}_{e}=\mathcal{H}_{e}^{\prime}$ if $q>2$ and $\mathcal{H}_{e} \neq \mathcal{H}_{e}^{\prime}$ if $q=2$, see De Bruyn [18].

In some of the above-mentioned cases, the set $\mathcal{H}_{e}^{\prime}$ coincides with the set of all hyperplanes of $\Delta$. By definition this is the case if $e$ is the Grassmannembedding of $D H\left(2 n-1, q^{2}\right)$ (Case (II)). It is never the case if $e$ is the spin-embedding of $D Q^{-}(2 n+1, q)$ (Case (IV)), see De Bruyn [14, Section 1.4]. It is the case for the spin-embedding of $D Q(2 n, q)$ (Case (III)) if and only if $q$ is odd. (By Payne and Thas [26], all hyperplanes of the quads 


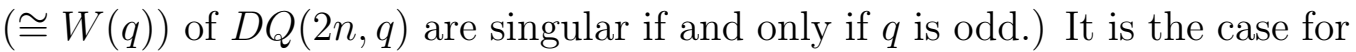
the Grassmann-embedding of the dual polar space $D W(2 n-1, q)$ if and only if all ovoids of the quads of $D W(2 n-1, q)$ are classical. As remarked above such quads are isomorphic to $Q(4, q)$. Now, all ovoids of $Q(4, q)$ are classical if $q$ is prime ([1]), $q=4$ ([2], [24]) or $q=16$ ([22], [23]). Non-classical ovoids of $Q(4, q)$ are known to exist for every $q=p^{h}$ where $p$ is an odd prime and $h \geq 2$ ([21], [27], [32]) and for every $q=2^{2 n+1}$ where $n \geq 1$ ([33]).

Definitions. If $H_{1}$ and $H_{2}$ are two distinct hyperplanes of $\mathcal{H}_{e}$, then $\left[H_{1}, H_{2}\right]$ denotes the set of all hyperplanes of $\Delta$ of the form $e^{-1}(e(P) \cap \Pi)$, where $\Pi$ is a hyperplane of $\Sigma$ through $\left\langle e\left(H_{1}\right)\right\rangle_{\Sigma} \cap\left\langle e\left(H_{2}\right)\right\rangle_{\Sigma}$. (Notice that $\left\langle e\left(H_{1}\right)\right\rangle_{\Sigma} \neq$ $\left\langle e\left(H_{2}\right)\right\rangle_{\Sigma}$, since $\left\langle e\left(H_{i}\right)\right\rangle_{\Sigma} \cap e(P)=e\left(H_{i}\right)$ for every $i \in\{1,2\}$.) We define $\left[H_{1}, H_{2}\right]^{\prime}:=\left[H_{1}, H_{2}\right] \backslash\left\{H_{1}, H_{2}\right\}$.

Let $Q$ be a quad of $\Delta$ and let $e_{Q}: Q \rightarrow\langle e(Q)\rangle_{\Sigma}$ be the projective embedding of $Q$ induced by $e$. Then the pair $\left(Q, e_{Q}\right)$ has the same type as $(\Delta, e)$ (i.e. (I), (II), (III) or (IV)). Let $\mathcal{H}_{Q}:=\mathcal{H}_{e_{Q}}$ denote the set of all hyperplanes of $Q$ arising from $e_{Q}$. If $G_{1}$ and $G_{2}$ are two distinct hyperplanes of $\mathcal{H}_{Q}$ and $x \in$ $Q \backslash\left(G_{1} \cup G_{2}\right)$, then we show in Section 2 that there exists a unique hyperplane $G_{x} \in \mathcal{H}_{Q}$ through $x$ satisfying $G_{1} \cap G_{x}=G_{1} \cap G_{2}=G_{2} \cap G_{x}$. Notice that $G_{x} \in\left[G_{1}, G_{2}\right]^{\prime}$ since there exists a hyperplane of $\left[G_{1}, G_{2}\right]^{\prime}$ satisfying that property.

Let $H_{1}$ and $H_{2}$ be two distinct hyperplanes of $\mathcal{H}_{e}$. We define a graph $\Gamma\left(H_{1}, H_{2}\right)$ with vertex set $P \backslash\left(H_{1} \cup H_{2}\right)$. Two vertices $x$ and $y$ are adjacent if one of the following two conditions holds:

(i) $\mathrm{d}(x, y)=1$ and the line $x y$ meets $H_{1} \cap H_{2}$;

(ii) $\mathrm{d}(x, y)=2,\langle x, y\rangle \cap H_{1} \neq\langle x, y\rangle \cap H_{2}$ and the unique element of $\left[\langle x, y\rangle \cap H_{1},\langle x, y\rangle \cap H_{2}\right]^{\prime}$ containing $x$ coincides with the unique element of $\left[\langle x, y\rangle \cap H_{1},\langle x, y\rangle \cap H_{2}\right]^{\prime}$ containing $y$.

Let $\mathcal{C}$ denote the set of all connected components of $\Gamma\left(H_{1}, H_{2}\right)$ and put

$$
\mathcal{H}:=\left\{C \cup\left(H_{1} \cap H_{2}\right) \mid C \in \mathcal{C}\right\} .
$$

The following is the main result of this paper. We will prove it in Section 4 .

Main Theorem. Every element of $\mathcal{H}$ is a hyperplane of $\Delta$. Moreover, the following statements are equivalent for a hyperplane $H$ of $\Delta$ :

(1) $H \in \mathcal{H}$;

(2) $H$ is a hyperplane of $\mathcal{H}_{e}^{\prime}$ satisfying $H \cap H_{1}=H_{1} \cap H_{2}=H_{2} \cap H$;

(3) $H \in\left[H_{1}, H_{2}\right]^{\prime}$. 
Remarks. (1) The conclusions of the Main Theorem are also valid if $e$ is the spin-embedding of the dual polar space $D Q(2 n, \mathbb{K}), n \geq 2$, where $\mathbb{K}$ is an arbitrary possibly infinite field (with the same proof). Since for the three other cases, we could not completely omit the finiteness assumption, we have chosen to state all results in the finite case.

(2) The conclusions of the Main Theorem were already known if $e$ is the spin-embedding of the dual polar space $D Q(2 n, \mathbb{K}), n \geq 2$, or the Grassmannembedding of the Hermitian dual polar space $D H\left(5, q^{2}\right)$, see respectively De Bruyn [14, Proposition 2.2] and De Bruyn \& Pralle [20, Section 4.2]. We have decided to include also these cases in our discussion, since we will give a unified treatment for the cases (I), (II), (III) and (IV).

\section{3 (Possible) Applications of the Main Theorem}

Consider one of the possibilities for $(\Delta, e)$ mentioned in Section 1.2. The set $\mathcal{H}_{e}$ carries the structure of a projective space isomorphic to $\Sigma$ if one takes the sets $\left[H_{1}, H_{2}\right]$ with $H_{1}, H_{2} \in \mathcal{H}_{e}$ and $H_{1} \neq H_{2}$ as lines. Suppose $\mathcal{G} \subseteq \mathcal{H}_{e}$ is a set of points of $\mathcal{H}_{e}$ which generates $\mathcal{H}_{e}$. In cases (II), (III) and (IV), one can take for $\mathcal{G}$ the set of all singular hyperplanes of $\Delta$, see Cardinali, De Bruyn and Pasini [7, Section 4]. In case (I) it is not always true that the set of all singular hyperplanes of $\Delta$ generates $\mathcal{H}_{e}$. (If $q$ is even, it is even never the case.)

We list three (possible) applications of the Main Theorem.

(1) If $H_{1}$ and $H_{2}$ are two given distinct elements of $\mathcal{H}_{e}$, then by the Main Theorem we can give a construction of all the hyperplanes of $\left[H_{1}, H_{2}\right]^{\prime}$ without implementing the embedding $e$ : these hyperplanes are precisely the sets $\left(H_{1} \cap H_{2}\right) \cup C$, where $C$ is a connected component of the graph $\Gamma\left(H_{1}, H_{2}\right)$. So, we obtain a direct construction of the hyperplanes of $\left[H_{1}, H_{2}\right]^{\prime}$, i.e. a construction which only refers to properties of the hyperplanes $H_{1}, H_{2}$ and the dual polar space $\Delta$ and not to properties of the embedding $e$ and its associated embedding space $\Sigma$.

(2) In practical applications it might be possible to classify certain hyperplanes $H \in \mathcal{H}_{e}^{\prime}$ by first constructing two hyperplanes $H_{1}, H_{2} \in \mathcal{H}_{e}$ and subsequently showing that $H \cap H_{1}=H_{1} \cap H_{2}=H_{2} \cap H$. This method was used in the paper [14] to show that all locally singular hyperplanes of $D Q(2 n, \mathbb{K})$ arise from its spin-embedding, and in the paper [20] (together with other techniques) to classify the hyperplanes of the dual polar space $\operatorname{DH}\left(5, q^{2}\right), q \neq 2$. 
(3) Suppose one wants to show that all hyperplanes of $\mathcal{H}_{e}$ satisfy a certain property (P). Then one can proceed as follows: (1) Show that all hyperplanes of $\mathcal{G}$ satisfy Property (P); (2) Show by using the explicit construction of the graph $\Gamma\left(H_{1}, H_{2}\right)$ that if two distinct hyperplanes $H_{1}$ and $H_{2}$ of $\mathcal{H}_{e}$ satisfy Property $(\mathrm{P})$, then also every member of $\left[H_{1}, H_{2}\right]^{\prime}$ satisfies Property $(\mathrm{P})$. This method was used in the paper [17] to show that all locally singular hyperplanes of $D Q(2 n, \mathbb{K})$ satisfy a certain property.

\section{A common property of the hyperplanes of some classical generalized quadrangles}

In this section, we will prove a common property of the hyperplanes of the classical generalized quadrangles $W(q)=W(3, q) \cong D Q(4, q), Q^{-}(5, q) \cong$ $D H\left(3, q^{2}\right), Q(4, q) \cong D W(3, q)$ and $H\left(3, q^{2}\right) \cong D Q^{-}(5, q)$.

Lemma 2.1 If $G_{1}$ and $G_{2}$ are two distinct singular hyperplanes of the generalized quadrangle $W(q)$, then through every point $x \in W(q) \backslash\left(G_{1} \cup G_{2}\right)$, there exists a unique singular hyperplane $G_{x}$ satisfying $G_{x} \cap G_{1}=G_{1} \cap G_{2}=$ $G_{2} \cap G_{x}$.

Proof. Let $W(q)$ be embedded in the projective space $\mathrm{PG}(3, q)$. We regard the singular hyperplanes of $W(q)$ as hyperplanes of $\mathrm{PG}(3, q)$. Then $G_{1} \cap G_{2}$ is a line of $\operatorname{PG}(3, q)$ which is either an ordinary line or a hyperbolic line of the symplectic generalized quadrangle $W(q)$. If $G_{x}$ is a singular hyperplane through $x$ satisfying $G_{x} \cap G_{1}=G_{1} \cap G_{2}=G_{2} \cap G_{x}$, then $G_{x}$ (regarded as hyperplane of $\mathrm{PG}(3, q))$ is necessarily equal to $\left\langle x, G_{1} \cap G_{2}\right\rangle$. This proves the lemma.

Lemma 2.2 If $G_{1}$ and $G_{2}$ are two distinct hyperplanes of the generalized quadrangle $Q^{-}(5, q)$, then through every point $x \in Q^{-}(5, q) \backslash\left(G_{1} \cup G_{2}\right)$, there exists a unique hyperplane $G_{x}$ satisfying $G_{x} \cap G_{1}=G_{1} \cap G_{2}=G_{2} \cap G_{x}$.

Proof. Let $Q^{-}(5, q)$ be embedded in the projective space $\operatorname{PG}(5, q)$. By Payne and Thas [26], every hyperplane of $Q^{-}(5, q)$ is either a singular hyperplane or a subquadrangle isomorphic to $Q(4, q)$. In each of the two cases, the hyperplane arises from a hyperplane of $\operatorname{PG}(5, q)$. Let $\Pi_{i}, i \in\{1,2\}$, denote the unique hyperplane of $\operatorname{PG}(5, q)$ such that $\Pi_{i} \cap Q^{-}(5, q)=G_{i}$. Since $\operatorname{dim}\left(\Pi_{1} \cap \Pi_{2}\right)=3$ and $Q^{-}(5, q)$ does not contain planes, $\Pi_{1} \cap \Pi_{2}$ intersects $Q^{-}(5, q)$ in one of the following: (i) an elliptic quadric $Q^{-}(3, q)$; (ii) a hyperbolic quadric $Q^{+}(3, q)$; (iii) a cone $p Q(2, q)$; (iv) a line $L$. Notice that $G_{1} \cap G_{2}$ 
is a hyperplane of both $G_{1}$ and $G_{2}$ (regarded as point-line geometries). So, if case (iv) occurs, then $G_{1}$ and $G_{2}$ are necessarily singular hyperplanes whose deepest points lie on $L$.

Suppose now that $G$ is a hyperplane of $Q^{-}(5, q)$ through $x$ satisfying $G \cap G_{1}=G_{1} \cap G_{2}=G_{2} \cap G$ and let $\Pi$ denote the unique hyperplane of $\operatorname{PG}(5, q)$ containing all the points of $G$. In cases (i), (ii) and (iii), $\Pi$ must contain the 3 -space $\Pi_{1} \cap \Pi_{2}$ and hence coincides with $\left\langle\Pi_{1} \cap \Pi_{2}, x\right\rangle$. In these cases, $G_{x}:=\left\langle\Pi_{1} \cap \Pi_{2}, x\right\rangle \cap Q^{-}(5, q)$ is the unique hyperplane of $Q^{-}(5, q)$ through $x$ satisfying $G_{x} \cap G_{1}=G_{1} \cap G_{2}=G_{2} \cap G_{x}$. Suppose case (iv) occurs. As remarked above, $G_{1}$ and $G_{2}$ are singular hyperplanes whose deepest points lie on $L$. Since $G \cap G_{1}=L$, also $G$ must be a singular hyperplane with deepest point on $L$. Since $x \in G, G$ must be the singular hyperplane with deepest point $\pi_{L}(x)$. From the uniqueness of $G$, it now follows that $G=\left\langle\Pi_{1} \cap \Pi_{2}, x\right\rangle \cap$ $Q^{-}(5, q)$. So, also in case (iv) $G_{x}:=\left\langle\Pi_{1} \cap \Pi_{2}, x\right\rangle \cap Q^{-}(5, q)$ is the unique hyperplane of $Q^{-}(5, q)$ through $x$ satisfying $G_{x} \cap G_{1}=G_{1} \cap G_{2}=G_{2} \cap G_{x}$.

Definition. By Payne and Thas [26], every hyperplane of the generalized quadrangle $Q(4, q)$ is either a singular hyperplane, a $(q+1) \times(q+1)$-subgrid or an ovoid. A hyperplane of $Q(4, q)$ is called classical if it is either a singular hyperplane, a $(q+1) \times(q+1)$-subgrid or a classical ovoid. The classical hyperplanes of $Q(4, q)$ are precisely those hyperplanes of $Q(4, q)$ which arise from an embedding.

Lemma 2.3 If $G_{1}$ and $G_{2}$ are two distinct classical hyperplanes of $Q(4, q)$, then through every point $x \in Q(4, q) \backslash\left(G_{1} \cup G_{2}\right)$, there exists a unique classical hyperplane $G_{x}$ through $x$ satisfying $G_{x} \cap G_{1}=G_{1} \cap G_{2}=G_{2} \cap G_{x}$.

Proof. Let $Q(4, q)$ be embedded in the projective space $\mathrm{PG}(4, q)$. Let $\Pi_{i}$, $i \in\{1,2\}$, be the unique hyperplane of $\mathrm{PG}(4, q)$ such that $G_{i}=\Pi_{i} \cap Q(4, q)$. Since $\operatorname{dim}\left(\Pi_{1} \cap \Pi_{2}\right)=2$ and $Q(4, q)$ does not contain planes, $\Pi_{1} \cap \Pi_{2}$ intersects $Q(4, q)$ in one of the following: (i) a point $p$; (ii) a line $L$; (iii) the union of two distinct lines; (iv) a nondegenerate conic. If case (i) occurs, then since $G_{1} \cap G_{2}$ is a hyperplane of both $G_{1}$ and $G_{2}$, there exists an $i \in\{1,2\}$ such that $G_{i}$ is a classical ovoid of $Q(4, q)$ containing $p$ and $G_{3-i}$ is either a classical ovoid of $Q(4, q)$ containing $p$ or the singular hyperplane of $Q(4, q)$ with deepest point $p$. If case (ii) occurs, then since $G_{1} \cap G_{2}$ is a hyperplane of both $G_{1}$ and $G_{2}$, $G_{1}$ and $G_{2}$ are necessarily singular hyperplanes with deepest points on $L$. Suppose now that $G$ is a classical hyperplane of $Q(4, q)$ through $x$ satisfying $G_{1} \cap G=G_{1} \cap G_{2}=G_{2} \cap G$ and let $\Pi$ denote the unique hyperplane of $\mathrm{PG}(4, q)$ containing $G$. 
If case (iii) or (iv) occurs, then $\Pi$ is necessarily equal to $\left\langle\Pi_{1} \cap \Pi_{2}, x\right\rangle$. It follows that $G_{x}:=\left\langle\Pi_{1} \cap \Pi_{2}, x\right\rangle \cap Q(4, q)$ is the unique classical hyperplane of $Q(4, q)$ satisfying $G_{x} \cap G_{1}=G_{1} \cap G_{2}=G_{2} \cap G_{x}$.

If case (i) occurs, then without loss of generality, we may suppose that $G_{1}$ is a classical ovoid of $Q(4, q)$ containing $p$. Since $G_{1} \cap G_{2}$ is a point, $\Pi_{1} \cap \Pi_{2}$ is the tangent hyperplane at the point $G_{1} \cap G_{2}$ of the elliptic quadric $\Pi_{1} \cap Q(4, q)$ of $\Pi_{1}$. Similarly, since $G \cap G_{1}=G_{1} \cap G_{2}$ is a point, $\Pi \cap \Pi_{1}$ must be the tangent hyperplane at the point $G_{1} \cap G_{2}$ of the elliptic quadric $\Pi_{1} \cap Q(4, q)$ of $\Pi_{1}$. Since $\Pi \cap \Pi_{1}=\Pi_{1} \cap \Pi_{2}$, we necessarily have $\Pi=\left\langle\Pi_{1} \cap \Pi_{2}, x\right\rangle$. Hence, $G_{x}:=\left\langle\Pi_{1} \cap \Pi_{2}, x\right\rangle \cap Q(4, q)$ is the unique classical hyperplane of $Q(4, q)$ satisfying $G_{x} \cap G_{1}=G_{1} \cap G_{2}=G_{2} \cap G_{x}$.

If case (ii) occurs with $G_{1} \cap G_{2}=L$, then $G_{1}$ and $G_{2}$ must be singular hyperplanes with deepest point on $L$. Since $G \cap G_{1}=G_{1} \cap G_{2}=L$, also $G$ must be a singular hyperplane with deepest point on $L$. Since $x \in G, G$ necessarily is the singular hyperplane of $Q(4, q)$ with deepest point $\pi_{L}(x)$. So, also in this case, there exists a unique classical hyperplane $G_{x}$ in $Q(4, q)$ satisfying $G_{x} \cap G_{1}=G_{1} \cap G_{2}=G_{2} \cap G_{x}$. This hyperplane $G_{x}$ coincides with $\left\langle\Pi_{1} \cap \Pi_{2}, x\right\rangle \cap Q(4, q)$.

Definition. By Payne and Thas [26], every hyperplane of the generalized quadrangle $H\left(3, q^{2}\right)$ is either a singular hyperplane or an ovoid. A hyperplane of $H\left(3, q^{2}\right)$ is called classical if it is either a singular hyperplane or a classical ovoid. The classical hyperplanes of $H\left(3, q^{2}\right)$ are precisely those hyperplanes of $H\left(3, q^{2}\right)$ which arise from an embedding.

Lemma 2.4 If $G_{1}$ and $G_{2}$ are two distinct classical hyperplanes of $H\left(3, q^{2}\right)$, then through every point $x \in H\left(3, q^{2}\right) \backslash\left(G_{1} \cup G_{2}\right)$, there exists a unique classical hyperplane $G_{x}$ satisfying $G_{x} \cap G_{1}=G_{1} \cap G_{2}=G_{2} \cap G_{x}$.

Proof. Let $H\left(3, q^{2}\right)$ be embedded in the projective space PG(3, $\left.q^{2}\right)$. Let $\Pi_{i}$, $i \in\{1,2\}$, be the unique plane of $\mathrm{PG}\left(3, q^{2}\right)$ such that $G_{i}=\Pi_{i} \cap H\left(3, q^{2}\right)$. Since $\operatorname{dim}\left(\Pi_{1} \cap \Pi_{2}\right)=1, \Pi_{1} \cap \Pi_{2}$ intersects $H\left(3, q^{2}\right)$ in either a point, a Baer subline of $\Pi_{1} \cap \Pi_{2}$ or the whole line $\Pi_{1} \cap \Pi_{2}$. If $\Pi_{1} \cap \Pi_{2} \cap H\left(3, q^{2}\right)$ is a point $p$, there exists an $i \in\{1,2\}$ such that $G_{i}$ is a classical ovoid of $H\left(3, q^{2}\right)$ containing $p$ and $G_{3-i}$ is either a classical ovoid of $H\left(3, q^{2}\right)$ containing $p$ or the singular hyperplane of $H\left(3, q^{2}\right)$ with deepest point $p$. Suppose now that $G$ is a classical hyperplane of $H\left(3, q^{2}\right)$ through $x$ satisfying $G_{1} \cap G=$ $G_{1} \cap G_{2}=G_{2} \cap G$ and let $\Pi$ denote the unique hyperplane of $\operatorname{PG}\left(3, q^{2}\right)$ such that $G=\Pi \cap H\left(3, q^{2}\right)$.

If $\Pi_{1} \cap \Pi_{2}$ intersects $H\left(3, q^{2}\right)$ in either a Baer subline of $\Pi_{1} \cap \Pi_{2}$ or the whole line $\Pi_{1} \cap \Pi_{2}$, then $\Pi$ necessarily coincides with $\left\langle\Pi_{1} \cap \Pi_{2}, x\right\rangle$. Hence, in 
these cases $G_{x}:=\left\langle\Pi_{1} \cap \Pi_{2}, x\right\rangle \cap H\left(3, q^{2}\right)$ is the unique classical hyperplane of $H\left(3, q^{2}\right)$ satisfying $G_{x} \cap G_{1}=G_{1} \cap G_{2}=G_{2} \cap G_{x}$.

Suppose $\Pi_{1} \cap \Pi_{2}$ intersects $H\left(3, q^{2}\right)$ in a point. Then without loss of generality, we may suppose that $G_{1}$ is a classical ovoid of $H\left(3, q^{2}\right)$. Since $G_{1} \cap G_{2}$ is a point, the line $\Pi_{1} \cap \Pi_{2}$ is the tangent line at the point $G_{1} \cap G_{2}$ of the unital $\Pi_{1} \cap H\left(3, q^{2}\right)$ of $\Pi_{1}$. Similarly, since $G \cap G_{1}=G_{1} \cap G_{2}$ is a point, $\Pi \cap \Pi_{1}$ must be the tangent line at the point $G_{1} \cap G_{2}$ of the unital $\Pi_{1} \cap H\left(3, q^{2}\right)$ of $\Pi_{1}$. Since $\Pi \cap \Pi_{1}=\Pi_{1} \cap \Pi_{2}$, we necessarily have $\Pi=\left\langle\Pi_{1} \cap \Pi_{2}, x\right\rangle$. Hence, $G_{x}:=\left\langle\Pi_{1} \cap \Pi_{2}, x\right\rangle \cap H\left(3, q^{2}\right)$ is the unique classical hyperplane of $H\left(3, q^{2}\right)$ satisfying $G_{x} \cap G_{1}=G_{1} \cap G_{2}=G_{2} \cap G_{x}$.

\section{The structure of the sets $\Lambda_{H}(x), x \in H$}

Recall that if $H$ is a hyperplane of a dual polar space $\Delta$ and if $x \in H$, then $\Lambda_{H}(x)$ denotes the set of lines through $x$ contained in $H$. We can regard $\Lambda_{H}(x)$ as a set of points of the projective space $\operatorname{Res}_{\Delta}(x)$.

Lemma 3.1 If $H$ is a hyperplane of the dual polar space $\Delta=D Q(2 n, q)$, $n \geq 2$, arising from its spin-embedding, then for every point $x$ of $H, \Lambda_{H}(x)$ is either a hyperplane of $\operatorname{Res}_{\Delta}(x)$ or the whole set of points of $\operatorname{Res}_{\Delta}(x)$.

Proof. Every hyperplane of $D Q(2 n, q), n \geq 2$, arising from its spinembedding is locally singular, see e.g. De Bruyn [14, Proposition 1.2]. Now, by Lemma 3.2 of Cardinali, De Bruyn and Pasini [6], if $x$ is a point of a locally singular hyperplane $H$ of $\Delta=D Q(2 n, q)$, then $\Lambda_{H}(x)$ is either a hyperplane of $\operatorname{Res}_{\Delta}(x)$ or the whole set of points of $\operatorname{Res}_{\Delta}(x)$.

For a proof of the following two lemmas (3.2 and 3.3), we refer to Cardinali and De Bruyn [5, Corollary 1.5] or Pasini [25, Theorem 9.3].

Lemma 3.2 If $H$ is a hyperplane of the dual polar space $\Delta=D W(2 n-1, q)$, $n \geq 2$, arising from its Grassmann-embedding, then for every point $x$ of $H$, $\Lambda_{H}(x)$ is a possibly degenerate quadric of $\operatorname{Res}_{\Delta}(x)$.

Remark. In Lemma 3.2, we have considered the whole point-set of $\operatorname{Res}_{\Delta}(x) \cong$ $\mathrm{PG}(n-1, q)$ as a degenerate quadric of $\operatorname{Res}_{\Delta}(x)$. There are indeed hyperplanes of $D W(2 n-1, q), n \geq 2$, arising from its Grassmann-embedding which have deep points, e.g. the singular hyperplanes of $D W(2 n-1, q)$.

Lemma 3.3 If $H$ is a hyperplane of the dual polar space $\Delta=D H\left(2 n-1, q^{2}\right)$, $n \geq 2$, arising from its Grassmann-embedding, then for every point $x$ of $H$, $\Lambda_{H}(x)$ is a possibly degenerate Hermitian variety of $\operatorname{Res}_{\Delta}(x)$. 
Remark. In Lemma 3.3, we have considered the whole point-set of $\operatorname{Res}_{\Delta}(x) \cong$ $\mathrm{PG}\left(n-1, q^{2}\right)$ as a degenerate Hermitian variety of $\operatorname{Res}_{\Delta}(x)$. There are indeed hyperplanes of $D H\left(2 n-1, q^{2}\right), n \geq 2$, arising from its Grassmann-embedding which have deep points, e.g. the singular hyperplanes of $D H\left(2 n-1, q^{2}\right)$.

We now prove the following lemma.

Lemma 3.4 If $H$ is a hyperplane of the dual polar space $\Delta=D Q^{-}(2 n+$ $1, q), n \geq 2$, arising from its spin-embedding, then for every point $x \in H$, $\Lambda_{H}(x)$ is a subspace of co-dimension at most 2 of $\operatorname{Res}_{\Delta}(x)$.

Proof. The proof is divided into several steps.

Step 1. $\Lambda_{H}(x)$ is a subspace of $\operatorname{Res}_{\Delta}(x)$.

Proof. Let $L_{1}$ and $L_{2}$ be two distinct lines through $x$ contained in $H$, and let $Q \cong H\left(3, q^{2}\right)$ be the quad $\left\langle L_{1}, L_{2}\right\rangle$. Since the generalized quadrangle $H\left(3, q^{2}\right)$ does not admit proper subquadrangles (see Payne and Thas [26]), $Q \cap H$ is either $Q$, an ovoid of $Q$ or a singular hyperplane of $Q$. Since $L_{1} \cup L_{2} \subseteq H$, there are two possibilities. Either $Q \subseteq H$ or $Q \cap H$ is the singular hyperplane of $Q$ with deepest point $x$. In either case, every line of $Q$ through $x$ is contained in $H$. This proves that $\Lambda_{H}(x)$ is a subspace of $\operatorname{Res}_{\Delta}(x)$.

Step 2. The lemma holds if $n=2$.

Proof. Obviously, every subspace of $\operatorname{Res}_{\Delta}(x) \cong \mathrm{PG}(1, q)$ has co-dimension at most 2 .

Step 3. The lemma holds if $n=3$.

Proof. By De Bruyn [14, Theorem 1.5], there are three types of hyperplanes in $D Q^{-}(7, q)$ which arise from its spin-embedding: the singular hyperplanes, the so-called extensions of the classical ovoids in the quads and the so-called hexagonal hyperplanes.

(1) Suppose $H$ is the singular hyperplane of $D Q^{-}(7, q)$ with deepest point $x$ and let $y$ be an arbitrary point of $H$. If $\mathrm{d}(x, y) \leq 1$, then $\Lambda_{H}(y)$ is the whole set of points of $\operatorname{Res}_{\Delta}(y)$. If $\mathrm{d}(x, y)=2$, then $\Lambda_{H}(y)$ is a hyperplane of $\operatorname{Res}_{\Delta}(y)$.

(2) Suppose $H$ is the extension of a classical ovoid $O$ in a quad $Q$ of $D Q^{-}(7, q)$. Then $H$ is the hyperplane $O \cup \Delta_{1}(O)$ of $\Delta$. Let $y$ be an arbitrary point of $H$. If $y \notin Q$, then the subspace $\Lambda_{H}(y)$ of $\operatorname{Res}_{\Delta}(y)$ has co-dimension 2. If $y \in Q \backslash O$, then $\Lambda_{H}(y)$ is a hyperplane of $\operatorname{Res}_{\Delta}(y)$ and if $y \in O$, then $\Lambda_{H}(y)$ coincides with the whole set of points of $\operatorname{Res}_{\Delta}(y)$.

(3) Let $Q(6, q)$ be a nonsingular parabolic quadric which is obtained by intersecting $Q^{-}(7, q)$ with a hyperplane of the ambient projective space of 
$Q^{-}(7, q)$. Let $\mathcal{G}$ be a set of generators (= maximal subspaces) of $Q(6, q)$ which defines a so-called hexagonal hyperplane $G$ of the dual polar space $D Q(6, q)$ associated with $Q(6, q)$. [The hyperplane $G$ of $D Q(6, q)$ satisfies the following properties (see Shult [29]): (i) every quad of $D Q(6, q)$ is singular with respect to $G$; (ii) the points and lines of $D Q(6, q)$ contained in $G$ define a split-Cayley hexagon $H(q)$ (see Van Maldeghem [34] for the definition of this generalized polygon); (iii) for every point $x \in G$, there exists a unique quad $Q$ of $D Q(6, q)$ through $x$ such that $x^{\perp} \cap G=x^{\perp} \cap Q$.] Let $\mathcal{G}^{\prime}$ denote the set of generators $\alpha$ of $Q^{-}(7, q)$ not contained in $Q(6, q)$ such that every generator of $Q(6, q)$ through $\alpha \cap Q(6, q)$ belongs to $\mathcal{G}$. Then by Pralle [28], $H:=\mathcal{G} \cup \mathcal{G}^{\prime}$ is a hyperplane of $D Q^{-}(7, q)$. We call $\mathcal{G} \cup \mathcal{G}^{\prime}$ a hexagonal hyperplane of $D Q^{-}(7, q)$.

Now, let $\alpha$ be an arbitrary point of $H$, i.e. a generator of $Q^{-}(7, q)$ belonging to $\mathcal{G} \cup \mathcal{G}^{\prime}$.

If $\alpha \in \mathcal{G}^{\prime}$, then the line of $D Q^{-}(7, q)$ corresponding with the line $\alpha \cap Q(6, q)$ of $Q^{-}(7, q)$ is completely contained in $H$. So, the subspace $\Lambda_{H}(\alpha)$ of $\operatorname{Res}_{\Delta}(\alpha)$ has co-dimension at least 2 . It has co-dimension precisely 2 since every point of $\alpha \backslash Q(6, q)$ corresponds with a quad of $D Q^{-}(7, q)$ which is ovoidal with respect to $H$, see Pralle [28, Section 5.2].

If $\alpha \in \mathcal{G}$, then by property (iii) mentioned above there exists a unique point $x \in \alpha$ such that every generator of $Q(6, q)$ which intersects $\alpha$ in a line through $x$ belongs to $\mathcal{G}$. Then every generator of $Q^{-}(7, q)$ which intersects $\alpha$ in a line through $x$ belongs to $H$, in other words, every line of $D Q^{-}(7, q)$ through $\alpha$ contained in the quad $Q_{x}$ of $D Q^{-}(7, q)$ corresponding with $x$ is contained in $H$. So, the subspace $\Lambda_{H}(\alpha)$ of $\operatorname{Res}_{\Delta}(\alpha)$ has co-dimension at most 1 . It has co-dimension precisely 1 , since a hexagonal hyperplane of $D Q^{-}(7, q)$ does not admit deep points, see Pralle [28, Section 5.2].

Step 4. The lemma holds if $n \geq 4$.

Proof. In view of Step 1, it suffices to show that every plane of $\operatorname{Res}_{\Delta}(x)$ has at least one point in common with $\Lambda_{H}(x)$. Now, a plane of $\operatorname{Res}_{\Delta}(x)$ corresponds with a hex through $x$ which by Step 3 contains at least one line through $x$ which is contained in $H$. [If $F$ is a hex, then the embedding of $F$ induced by the spin-embedding of $\Delta$ is isomorphic to the spin-embedding of $F$. So, if $F$ is not contained in $H$, then the hyperplane $H \cap F$ of $F$ arises from the spin-embedding of $F$.]

\section{Proof of the Main Theorem}

Let $(\Delta, e)$ be as in (I), (II), (III) or (IV) of Section 1.2. Put $N_{e}:=3$ if case (II) or (III) occurs. Put $N_{e}:=5$ if case (I) or (IV) occurs. 
Definition. A point $x$ of a hyperplane $H$ of $\Delta$ is called nice (with respect to $H$ ) if the following property is satisfied:

- Case (I): $\Lambda_{H}(x)$ is a possibly degenerate quadric of $\operatorname{Res}_{\Delta}(x)$;

- Case (II): $\Lambda_{H}(x)$ is a possibly degenerate Hermitian variety of $\operatorname{Res}_{\Delta}(x)$;

- Case (III): $\Lambda_{H}(x)$ is a subspace of co-dimension at most 1 of $\operatorname{Res}_{\Delta}(x)$;

- Case (IV): $\Lambda_{H}(x)$ is a subspace of co-dimension at most 2 of $\operatorname{Res}_{\Delta}(x)$.

Lemma 4.1 Let $F$ be a convex subspace of diameter $\delta \geq 2$ of $\Delta$ and let $e_{F}: F \rightarrow\langle e(F)\rangle_{\Sigma}$ denote the embedding of $F$ induced by e. Then $\left(F, e_{F}\right)$ is of the same type (i.e., (I), (II), (III) or $(I V)$ ) as $(\Delta, e)$. Let $H$ be a hyperplane of $\Delta$ such that $F \nsubseteq H$, and let $x$ be a point of $H \cap F$ which is nice with respect to $H$. Then $x$ is also nice with respect to the hyperplane $F \cap H$ of $F$.

Proof. With $F$ there corresponds a subspace $\pi_{F}$ of dimension $\delta-1$ of $\operatorname{Res}_{\Delta}(x)$. The lemma is a straightforward corollary of the fact that $\pi_{F}$ intersects every possibly degenerate quadric (possibly degenerate Hermitian variety, respectively subspace of co-dimension at most $\mu$ ) of $\operatorname{Res}_{\Delta}(x)$ in a possibly degenerate quadric (possibly degenerate Hermitian variety, respectively subspace of co-dimension at most $\mu$ ) of $\pi_{F}$.

We leave the proof of the following lemma as a straightforward exercise to the reader.

Lemma 4.2 Let $x$ and $y$ be two points of $\Delta$ at maximal distance $n$ from each other. For every convex subspace $F$ of diameter $\delta$ through $x$, let $\theta(F)$ denote the unique convex subspace of diameter $n-\delta$ through $y$ which intersects $F$ in a unique point. Then $\theta$ defines an isomorphism between the projective space $\operatorname{Res}_{\Delta}(x)$ and the dual of the projective space $\operatorname{Res}_{\Delta}(y)$.

Lemma 4.3 Let $H$ be a hyperplane of $\Delta$ and let $x$ and $y$ be two points of $H$ at distance $\delta \geq N_{e}$ from each other which are nice with respect to $H$. Then there exists a line $L_{x} \subseteq H \cap\langle x, y\rangle$ through $x$ and a line $L_{y} \subseteq H \cap\langle x, y\rangle$ through $y$ such that $L_{x} \| L_{y}$ and $d\left(L_{x}, L_{y}\right)=\delta-1$.

Proof. Put $k:=2$ if $N_{e}=3$ and $k:=3$ if $N_{e}=5$. By Lemma 4.1 and the fact that $x$ is nice with respect to $H$, there exist $k$ lines $L_{1}, L_{2}, \ldots, L_{k}$ through $x$ which are contained in $\langle x, y\rangle \cap H$ and for which $\operatorname{diam}\left(\left\langle L_{1}, L_{2}, \ldots, L_{k}\right\rangle\right)=k$. Let $y^{\prime}$ denote the unique point of $\left\langle L_{1}, L_{2}, \ldots, L_{k}\right\rangle$ nearest to $y$. Then $\left\langle y, y^{\prime}\right\rangle$ has diameter $\delta-k$. Again by Lemma 4.1 and the fact that $y$ is nice with 
respect to $H$, there exists a line $L_{y}$ through $y$ contained in $\langle x, y\rangle \cap H$, but not in $\left\langle y, y^{\prime}\right\rangle$. For every $i \in\{1, \ldots, k\}$, let $F_{i}$ denote the unique max of $\langle x, y\rangle$ through $y$ meeting $L_{i}$. Then $F_{1} \cap F_{2} \cap \cdots \cap F_{k}=\left\langle y, y^{\prime}\right\rangle$ by Lemma 4.2. So, there exists an $i \in\{1, \ldots, k\}$ such that $F_{i}$ does not contain $L_{y}$. For such an $i$, put $L_{x}:=L_{i}$. Then $L_{x}$ and $L_{y}$ are parallel lines at distance $\delta-1$ from each other.

Now, let $H_{1}$ and $H_{2}$ be two distinct hyperplanes of $\mathcal{H}_{e}$. Define the graph $\Gamma:=\Gamma\left(H_{1}, H_{2}\right)$ as in Section 1.2. Let $\mathcal{C}$ denote the set of all connected components of $\Gamma$ and put

$$
\mathcal{H}:=\left\{C \cup\left(H_{1} \cap H_{2}\right) \mid C \in \mathcal{C}\right\} .
$$

Lemma 4.4 If $H$ is a hyperplane of $\Delta$ such that $H \cap H_{1}=H_{1} \cap H_{2}=H_{2} \cap H$, then every point $x \in H \backslash\left(H_{1} \cap H_{2}\right)$ is nice with respect to $H$.

Proof. Since $\left\langle e\left(H_{i}\right)\right\rangle_{\Sigma} \cap e(P)=e\left(H_{i}\right)$ for every $i \in\{1,2\},\left\langle e\left(H_{1}\right)\right\rangle_{\Sigma}$ and $\left\langle e\left(H_{2}\right)\right\rangle_{\Sigma}$ are two distinct hyperplanes of $\Sigma$ and $\left\langle e\left(H_{1}\right)\right\rangle_{\Sigma} \cap\left\langle e\left(H_{2}\right)\right\rangle_{\Sigma}$ does not contain the point $e(x)$. Let $\Pi$ be the hyperplane $\left\langle\left\langle e\left(H_{1}\right)\right\rangle_{\Sigma} \cap\left\langle e\left(H_{2}\right)\right\rangle_{\Sigma}, e(x)\right\rangle_{\Sigma}$ of $\Sigma$ and let $H^{\prime}$ be the hyperplane $e^{-1}(\Pi \cap e(P))$ of $\Delta$. Then $x \in H^{\prime}$ and $H^{\prime} \cap H_{1}=H_{1} \cap H_{2}=H_{2} \cap H^{\prime}$. The point $x$ is nice with respect to $H^{\prime}$ by Lemmas 3.1, 3.2, 3.3 and 3.4. Now, a line through $x$ is contained in $H$ if and only if it contains a point of $H \cap H_{1}=H_{1} \cap H_{2}$. Similarly, a line through $x$ is contained in $H^{\prime}$ if and only if it contains a point of $H^{\prime} \cap H_{1}=H_{1} \cap H_{2}$. It follows that $\Lambda_{H}(x)=\Lambda_{H^{\prime}}(x)$. Hence, $x$ is also nice with respect to $H$.

Lemma 4.5 If $H$ is a hyperplane of $\mathcal{H}_{e}^{\prime}$ satisfying $H \cap H_{1}=H_{1} \cap H_{2}=$ $\mathrm{H}_{2} \cap H$, then $\mathrm{H}$ belongs to $\mathcal{H}$.

Proof. Since $H$ is maximal subspace of $\Delta$ and $H_{1} \cap H_{2} \subseteq H$ is not a maximal subspace of $\Delta\left(H_{1} \cap H_{2}\right.$ is properly contained in the maximal subspaces $H_{1}$ and $\left.H_{2}\right)$, there exists a point $x^{*} \in H \backslash\left(H_{1} \cup H_{2}\right)$. Let $C$ denote the unique element of $\mathcal{C}$ containing $x^{*}$. We will prove that $H=C \cup\left(H_{1} \cap H_{2}\right) \in \mathcal{H}$.

Step 1. $C \cup\left(H_{1} \cap H_{2}\right) \subseteq H$.

Proof. In view of the facts that $x^{*} \in C \cap H$ and $H_{1} \cap H_{2} \subseteq H$, we need to show the following: if $x \in H \backslash\left(H_{1} \cap H_{2}\right)$ and $y$ is a vertex of $\Gamma$ adjacent to $x$, then also $y \in H$. We distinguish two cases:

Suppose $\mathrm{d}(x, y)=1$. Then the line $x y$ meets $H_{1} \cap H_{2}=H \cap H_{1}$. Since $H$ is a subspace, $x y \subseteq H$. Hence, $y \in H$.

Suppose $\mathrm{d}(x, y)=2$. Let $Q$ be the unique quad through the points $x$ and $y$. Then $Q \cap H_{1} \neq Q \cap H_{2}$ and $G_{x}=G_{y}$, where $G_{x}$ (respectively $G_{y}$ ) denotes the unique element of $\left[Q \cap H_{1}, Q \cap H_{2}\right]^{\prime}$ containing $x$ (respectively $y$ ). Since 
$H \cap H_{1}=H_{1} \cap H_{2}=H_{2} \cap H,(Q \cap H) \cap\left(Q \cap H_{1}\right)=\left(Q \cap H_{1}\right) \cap\left(Q \cap H_{2}\right)=$ $\left(Q \cap H_{2}\right) \cap(Q \cap H)$. Since $H_{1}, H_{2} \in \mathcal{H}_{e}, H_{1} \cap Q$ and $H_{2} \cap Q$ belong to $\mathcal{H}_{Q}:=\mathcal{H}_{e_{Q}}$. Since $H \in \mathcal{H}_{e}^{\prime}$, also $H \cap Q$ belongs to $\mathcal{H}_{Q}$. By Lemmas 2.1, 2.2, 2.3 and 2.4, $Q \cap H \in\left[Q \cap H_{1}, Q \cap H_{2}\right]^{\prime}$. From $x \in Q \cap H$, it then follows that $Q \cap H=G_{x}=G_{y}$. Hence, $y \in H$.

Step 2. $H \subseteq C \cup\left(H_{1} \cap H_{2}\right)$.

Proof. We will prove the following by induction on $i \geq 1$ : if $x, y \in H \backslash$ $\left(H_{1} \cap H_{2}\right)$ with $\mathrm{d}(x, y)=i$ and $x \in C$, then also $y \in C$. The property then immediately follows from the fact that $x^{*} \in H \cap C$.

(i) If $\mathrm{d}(x, y)=1$, then the line $x y$ meets $H \cap H_{1}=H_{1} \cap H_{2}$. Hence, $x$ and $y$ are adjacent points of $\Gamma$ and $y \in C$.

(ii) Suppose d $(x, y)=2$ and consider the quad $Q:=\langle x, y\rangle$. If $Q \cap H_{1}=$ $Q \cap H_{2}$, then since $Q \cap H_{1}=Q \cap H_{2}$ is a maximal subspace of $Q$ and $x \in C \cap\left(Q \backslash\left(Q \cap H_{1}\right)\right)$, also $y \in C$ by successive application of (i). Suppose therefore that $Q \cap H_{1} \neq Q \cap H_{2}$. As above, the fact that $H_{1} \cap H=H_{1} \cap H_{2}=$ $H \cap H_{2}$ implies that $Q \cap H \in\left[Q \cap H_{1}, Q \cap H_{2}\right]^{\prime}$. Since $x, y \in Q \cap H$ and $x \in C$, also $y \in C$.

(iii) Suppose that there exists a line $L_{x} \subseteq H \cap\langle x, y\rangle$ through $x$ and a line $L_{y} \subseteq H \cap\langle x, y\rangle$ through $y$ such that every point of $L_{x}$ has distance $\mathrm{d}(x, y)-1$ from $L_{y}$. (Then $L_{x} \| L_{y}$.) Let $x^{\prime}$, respectively $y^{\prime}$, denote the unique point of $L_{x}$, respectively $L_{y}$, contained in $H \cap H_{1}=H_{1} \cap H_{2}$. Since $\left|L_{x}\right|,\left|L_{y}\right| \geq 3$, there exists a point $x^{\prime \prime} \in L_{x} \backslash\left\{x^{\prime}\right\}$ and a point $y^{\prime \prime} \in L_{y} \backslash\left\{y^{\prime}\right\}$ such that $\mathrm{d}\left(x^{\prime \prime}, y^{\prime \prime}\right)=\mathrm{d}(x, y)-1$. Since $x \in C, x^{\prime \prime} \in C$ by (i). By the induction hypothesis, it then follows that $y^{\prime \prime} \in C$. By (i), we finally obtain that $y \in C$.

In the sequel, we suppose that $\mathrm{d}(x, y) \geq 3$ and that there exists no pair ( $L_{x}, L_{y}$ ) of lines satisfying (i) $x \in L_{x} \subseteq H \cap\langle x, y\rangle$; (ii) $y \in L_{y} \subseteq\langle x, y\rangle \cap H$; (iii) every point of $L_{x}$ has distance $\mathrm{d}(x, y)-1$ from $L_{y}$. By Lemmas 4.3 and 4.4, this implies that $\mathrm{d}(x, y)<N_{e}$. So, we have that $\mathrm{d}(x, y) \in\{3,4\}$, $n \geq 3$ and $e$ is either the Grassmann-embedding of the dual polar space $D W(2 n-1, q)$ or the spin-embedding of the dual polar space $D Q^{-}(2 n+1, q)$.

(iv) Suppose first that $\mathrm{d}(x, y)=3$. If $L$ is a line through $y$ contained in $\langle x, y\rangle \cap H$, then since case (iii) does not occur, every line through $x$ contained in $\langle x, y\rangle \cap H$ is necessarily contained in the unique quad through $x$ meeting $L$. If $L_{1}$ and $L_{2}$ are two lines through $y$ contained in $\langle x, y\rangle \cap H$, then since case (iii) does not occur, every line through $x$ contained in $\langle x, y\rangle \cap H$ necessarily coincides with the unique line through $x$ meeting the quad $\left\langle L_{1}, L_{2}\right\rangle$. [By Lemma 4.2, this line coincides with the intersection of the two quads through $x$ which meet either $L_{1}$ or $L_{2}$.] So, by Lemmas 4.1 and 4.4 , there are three possibilities: 
(a) $x^{\perp} \cap\langle x, y\rangle \cap H$ is a line $L_{x}$ through $x$ and $\langle x, y\rangle \cap y^{\perp} \cap H$ is a line $L_{y}$ through $y$. Moreover, $\mathrm{d}\left(L_{x}, L_{y}\right)=1$.

(b) $x^{\perp} \cap\langle x, y\rangle \cap H$ is a line $L_{x}$ through $x$ and $\langle x, y\rangle \cap y^{\perp} \cap H=y^{\perp} \cap Q_{y}$, where $Q_{y}$ is the unique quad through $y$ meeting $L_{x}$ in a point.

(c) $y^{\perp} \cap\langle x, y\rangle \cap H$ is a line $L_{y}$ through $y$ and $\langle x, y\rangle \cap x^{\perp} \cap H=x^{\perp} \cap Q_{x}$, where $Q_{x}$ is the unique quad through $x$ meeting $L_{y}$ in a point.

We first treat case (b). Put $\left\{x^{\prime}\right\}=L_{x} \cap Q_{y}$. Since $x^{\prime} \in H$ and $y^{\perp} \cap Q_{y} \subseteq$ $H, Q_{y} \subseteq H$. If there exists a point $z \in\left(x^{\perp} \cap Q_{y}\right) \backslash\left(H_{1} \cap H_{2}\right)$, then by (i) and (ii) above, $z \in C$ and $y \in C$ since $\mathrm{d}(x, z) \leq 2$ and $\mathrm{d}(z, y) \leq 2$. So, we may suppose that $x^{\perp} \cap Q_{y} \subseteq H_{1} \cap H_{2}$. Since $x^{\perp} \cap\langle x, y\rangle \cap H$ is the line $L_{x}, H \cap\langle x, y\rangle$ is not the singular hyperplane of $\langle x, y\rangle$ with deepest point $x^{\prime}$. Hence, there exists a point $z^{\prime} \in\langle x, y\rangle \cap \Delta_{3}\left(x^{\prime}\right) \cap H$. [Otherwise, $H \cap\langle x, y\rangle \subseteq \Delta_{\leq 2}\left(x^{\prime}\right) \cap\langle x, y\rangle$ and this would imply that $H \cap\langle x, y\rangle=\Delta_{\leq 2}\left(x^{\prime}\right) \cap\langle x, y\rangle$ since $H \cap\langle x, y\rangle$ is a maximal subspace of $\langle x, y\rangle$.] The point $z:=\pi_{Q_{y}}\left(z^{\prime}\right)$ has distance 2 from $x^{\prime}$ and distance 3 from $x$. Since $H_{1} \cap H_{2} \cap Q_{y}$ is a proper subspace of $Q_{y}$ (recall $y \notin H_{1} \cap H_{2}$ ) containing the maximal subspace $x^{\perp \perp} \cap Q_{y}$ of $Q_{y}$, $H_{1} \cap H_{2} \cap Q_{y}=x^{\perp} \cap Q_{y}$. So, $z \in H \backslash\left(H_{1} \cap H_{2}\right)$. Since $z z^{\prime} \subseteq H$ and $z z^{\prime} \| L_{x}$, it follows that $z \in C$ by (iii). So, also $y \in C$ by either (i) or (ii).

Next, we treat case (c). Put $\left\{y^{\prime}\right\}=L_{y} \cap Q_{x}$. Since $y^{\prime} \in H$ and $x^{\perp} \cap Q_{x} \subseteq$ $H, Q_{x} \subseteq H$. If there exists a point $z \in\left(y^{\perp} \cap Q_{x}\right) \backslash\left(H_{1} \cap H_{2}\right)$, then by (i) and (ii) above, $z \in C$ and $y \in C$ since $\mathrm{d}(x, z) \leq 2$ and $\mathrm{d}(z, y) \leq 2$. So, we may suppose that $y^{\prime \perp} \cap Q_{x} \subseteq H_{1} \cap H_{2}$. Since $y^{\perp} \cap\langle x, y\rangle \cap H$ is a line $L_{y}, H \cap\langle x, y\rangle$ is not the singular hyperplane of $\langle x, y\rangle$ with deepest point $y^{\prime}$. Hence, there exists a point $z^{\prime} \in\langle x, y\rangle \cap \Delta_{3}\left(y^{\prime}\right) \cap H$. The point $z:=\pi_{Q_{x}}\left(z^{\prime}\right)$ has distance 2 from $y^{\prime}$ and distance 3 from $y$. Moreover, $z \notin H_{1} \cup H_{2}$. Since $x \in C$, also $z \in C$ by either (i) or (ii). Since $z z^{\prime} \subseteq H$ and $z z^{\prime} \| L_{y}$, it follows that $y \in C$ by (iii).

Finally, we treat case (a). Let $x^{\prime}$ and $y^{\prime}$ be the unique points of $L_{x}$ and $L_{y}$, respectively, such that $\mathrm{d}\left(x^{\prime}, y^{\prime}\right)=1$. Notice that $x^{\prime} y^{\prime} \subseteq H$. If $x^{\prime} \notin H_{1} \cap H_{2}$, then $x^{\prime} \in C$ and $y \in C$ since $\mathrm{d}\left(x, x^{\prime}\right)=1$ and $\mathrm{d}\left(x^{\prime}, y\right)=2$. Similarly, if $y^{\prime} \notin H_{1} \cap H_{2}$, then $y^{\prime} \in C$ and $y \in C$ since $\mathrm{d}\left(x, y^{\prime}\right)=2$ and $\mathrm{d}\left(y^{\prime}, y\right)=1$. So, we may suppose that $x^{\prime}, y^{\prime} \in H_{1} \cap H_{2}$. Then $L_{x} \backslash\left\{x^{\prime}\right\} \subseteq H \backslash\left(H_{1} \cap H_{2}\right)$ and $L_{y} \backslash\left\{y^{\prime}\right\} \subseteq H \backslash\left(H_{1} \cap H_{2}\right)$. Since $y^{\perp} \cap\langle x, y\rangle \cap H=L_{y}$, the quad $\left\langle x^{\prime}, y\right\rangle$ is singular with respect to $H$ with deepest point $y^{\prime}$. Now, let $L$ be a line of $\left\langle x^{\prime}, y\right\rangle$ through $x^{\prime}$ different from $x^{\prime} y^{\prime}$. Then $L$ is not contained in $H$. Since $x^{\perp} \cap\langle x, y\rangle \cap H=L_{x}$, the quad $\langle x, L\rangle$ is singular with respect to $H$. Let $u$ denote its deepest point. Since $x^{\perp} \cap\langle x, y\rangle \cap H=L_{x}$ and $L \nsubseteq H$, $u \in x x^{\prime} \backslash\left\{x, x^{\prime}\right\}$. By (i), $u \in C$. Since $u^{\perp} \cap\langle x, y\rangle \cap H$ is the union of at least 2 lines through $u$, one of the previous cases applies and we can conclude that $y \in C$. 
(v) Suppose now that $\mathrm{d}(x, y)=4$. If $L_{1}, L_{2}$ and $L_{3}$ are 3 lines through $x$ contained in $\langle x, y\rangle \cap H$ such that $\left\langle L_{1}, L_{2}, L_{3}\right\rangle$ is a hex, then since case (iii) does not occur, every line through $y$ contained in $\langle x, y\rangle \cap H$ is contained in $F_{1} \cap F_{2} \cap F_{3}$, where $F_{i}, i \in\{1,2,3\}$ is the unique hex through $y$ meeting $L_{i}$. By Lemma 4.2, $F_{1} \cap F_{2} \cap F_{3}$ is the unique line through $y$ meeting the hex $\left\langle L_{1}, L_{2}, L_{3}\right\rangle$. So, $y^{\perp} \cap\langle x, y\rangle \cap H$ is contained in the line $F_{1} \cap F_{2} \cap F_{3}$. This contradicts Lemmas 4.1 and 4.4. Hence, there exists a quad $Q_{x} \subseteq\langle x, y\rangle$ through $x$ such that $x^{\perp} \cap\langle x, y\rangle \cap H \subseteq Q_{x} \cap x^{\perp}$. By Lemmas 4.1 and 4.4, it then follows that $x^{\perp} \cap\langle x, y\rangle \cap H=Q_{x} \cap x^{\perp}$. Similarly, there exists a quad $Q_{y}$ through $y$ such that $y^{\perp} \cap\langle x, y\rangle \cap H=y^{\perp} \cap Q_{y}$. Since case (iii) does not occur, $Q_{x}$ and $Q_{y}$ intersect in a unique point $z$. Let $L$ be an arbitrary line of $Q_{x}$ through $x$. Then $L$ contains a unique point of $H \cap H_{1}=H_{1} \cap H_{2}$. Let $x^{\prime}$ denote a point of $L \backslash\{x\}$ not contained in $H_{1} \cap H_{2}$. Then $x^{\prime} \in C$ by (i). If $\mathrm{d}\left(x^{\prime}, y\right)=3$, then $y \in C$ by (iii) or (iv). So, we may suppose that $\mathrm{d}\left(x^{\prime}, y\right)=4$. Then $Q_{x}$ is the unique quad through $x^{\prime}$ meeting $Q_{y}$ (necessarily in the point $z)$. If $x^{\perp} \cap\langle x, y\rangle \cap H \neq x^{\perp} \cap Q_{x}$, then by the above discussion $y \in C$. Suppose therefore that $x^{\perp} \cap Q_{x}=x^{\perp} \cap\langle x, y\rangle \cap H$. Since $x^{\perp} \cap Q_{x} \subseteq H$ and $x^{\perp} \cap Q_{x} \subseteq H, Q_{x} \subseteq H$. In particular, we have $z \in H$. Since also $y^{\perp} \cap Q_{y} \subseteq H, Q_{y} \subseteq H$. If there exists a point $u \in\left(z^{\perp} \cap Q_{y}\right) \backslash\left(H_{1} \cap H_{2}\right)$, then $u \in C$ and $y \in C$ since $x \in C, \mathrm{~d}(x, u) \leq 3$ and $\mathrm{d}(u, y) \leq 2$. So, we may assume that $z^{\perp} \cap Q_{y} \subseteq H_{1} \cap H_{2}$. A similar reasoning shows that we may assume that $z^{\perp} \cap Q_{x} \subseteq H_{1} \cap H_{2}$. Now, let $R$ be a quad of $\langle x, y\rangle$ through $x$ such that $R \cap Q_{x}=\{x\}$. Then $R$ is ovoidal with respect to $H$, since $x^{\perp} \cap\langle x, y\rangle \cap H=x^{\perp} \cap Q_{x}$. In other words, $R \cap H$ is an ovoid of $R$.

Suppose $R \cap H \backslash\{x\} \subseteq H_{1} \cap H_{2}$. If $M$ is a line of $R$ contained in $H_{i}(i \in$ $\{1,2\})$, then $x \notin M$ and any line of $R$ through a point of $M \backslash\left(H \cup\left\{\pi_{M}(x)\right\}\right)$ is contained in $H_{i}$ as well as any line of $R$ through $\pi_{M}(x)$ distinct from $x \pi_{M}(x)$. So, $R \subseteq H_{i}$, in contradiction with the fact that $x \notin H_{i}$. Hence, $R \cap H_{i}$ does not contain lines. So, $R \cap H_{1}$ and $R \cap H_{2}$ are ovoids of $R$ containing $R \cap H \backslash\{x\}$. This would however imply that $R \cap H_{1}=R \cap H_{2}=R \cap H$, which is in contradiction with the fact that $x \notin H_{1} \cap H_{2}$.

Hence, there exists a point $v \in R \cap H \backslash\{x\}$ not belonging to $H_{1} \cap H_{2}$. By (ii), $v \in C$. Now, $\mathrm{d}(v, z)=\mathrm{d}\left(v, \pi_{Q_{x}}(v)\right)+\mathrm{d}\left(\pi_{Q_{x}}(v), z\right)=\mathrm{d}(v, x)+\mathrm{d}(x, z)=$ $2+2=4$. On the other hand, since $4=\mathrm{d}(v, z)=\mathrm{d}\left(v, \pi_{Q_{y}}(v)\right)+\mathrm{d}\left(\pi_{Q_{y}}(v), z\right)$, we have $\mathrm{d}\left(v, Q_{y}\right)=2$ and $v^{\prime}:=\pi_{Q_{y}}(v)$ lies at distance 2 from $z$. By (ii), $v^{\prime} \in C$. (Notice that $v^{\prime} \notin H_{1} \cap H_{2}$ since $z^{\perp} \cap Q_{y} \subseteq H_{1} \cap H_{2}$ is a maximal subspace of $Q_{y}$ and $y \notin H_{1} \cap H_{2}$.) By (i) or (ii), it then follows that $y \in C$.

We can now prove the Main Theorem. Obviously, (3) implies (2) and by Lemma 4.5, (2) implies (1). 
Now, let $H$ be an arbitrary element of $\mathcal{H}$. Let $x$ be an arbitrary point of $H \backslash\left(H_{1} \cap H_{2}\right)$. Then $H=\left(H_{1} \cap H_{2}\right) \cup C$, where $C$ is the connected component of $\Gamma$ containing $x$. Let $H^{\prime}$ denote the unique hyperplane of $\left[H_{1}, H_{2}\right]^{\prime}$ containing $x$. Then since $(3) \Rightarrow(2) \Rightarrow(1), H^{\prime} \in \mathcal{H}$. Since $x \in H^{\prime}$, we have $H^{\prime}=\left(H_{1} \cap H_{2}\right) \cup C=H$. Hence, $H=H^{\prime} \in\left[H_{1}, H_{2}\right]^{\prime}$. So, (1) also implies (3) and every element of $\mathcal{H}$ is a hyperplane of $\Delta$.

Note. Very recently, see [19], it was shown that the conclusion of Lemma 3.4 is also valid for infinite dual polar spaces of type $D Q^{-}(2 n+1, \mathbb{K})$. The conclusion of Lemma 2.4 is however not necessarily valid in the infinite case due to counterexamples. The reasoning given in and after the proof of Lemma 4.5 then allows us to draw the following conclusion: if $H_{1}$ and $H_{2}$ are two distinct hyperplanes of $D Q^{-}(2 n+1, \mathbb{K})$ arising from its spin-embedding and $\mathcal{H}$ is as defined in Section 1.2, then $\mathcal{H}=\left[H_{1}, H_{2}\right]^{\prime}$.

\section{References}

[1] S. Ball, P. Govaerts and L. Storme. On ovoids of parabolic quadrics. Des. Codes Cryptogr. 38 (2006), 131-145.

[2] A. Barlotti. Un'estensione del teorema di Segre-Kustaanheimo. Boll. Un. Mat. Ital. 10 (1955), 96-98.

[3] F. Buekenhout and P. J. Cameron. Projective and affine geometry over division rings. Handbook of Incidence Geometry, 27-62, North-Holland, Amsterdam, 1995.

[4] P. J. Cameron. Dual polar spaces. Geom. Dedicata 12 (1982), 75-85.

[5] I. Cardinali and B. De Bruyn. The structure of full polarized embeddings of symplectic and Hermitian dual polar spaces. Adv. Geom. 8 (2008), 111-137.

[6] I. Cardinali, B. De Bruyn and A. Pasini. Locally singular hyperplanes in thick dual polar spaces of rank 4. J. Combin. Theory Ser. A 113 (2006), 636-646.

[7] I. Cardinali, B. De Bruyn and A. Pasini. Minimal full polarized embeddings of dual polar spaces. J. Algebraic Combin. 25 (2007), 7-23.

[8] I. Cardinali, B. De Bruyn and A. Pasini. On the simple connectedness of hyperplane complements in dual polar spaces. Discrete Mathematics, to appear. 
[9] C. C. Chevalley. The algebraic theory of spinors. Columbia University Press, New York, 1954.

[10] B. N. Cooperstein. On the generation of dual polar spaces of unitary type over finite fields. European J. Combin. 18 (1997), 849-856.

[11] B. N. Cooperstein. On the generation of dual polar spaces of symplectic type over finite fields. J. Combin. Theory Ser. A 83 (1998), 221-232.

[12] B. N. Cooperstein and E. E. Shult. A note on embedding and generating dual polar spaces. Adv. Geom. 1 (2001), 37-48.

[13] B. De Bruyn. Near polygons. Birkhäuser, Basel, 2006.

[14] B. De Bruyn. The hyperplanes of $D Q(2 n, \mathbb{K})$ and $D Q^{-}(2 n+1, q)$ arising from their spin-embeddings. J. Combin. Theory Ser. A, 114 (2007), 681691.

[15] B. De Bruyn. A decomposition of the natural embedding spaces for the symplectic dual polar spaces. Linear Algebra Appl. 426 (2007), 462-477.

[16] B. De Bruyn. On the Grassmann-embeddings of the Hermitian dual polar spaces. Linear Multilinear Algebra, to appear.

[17] B. De Bruyn. A property of isometric mappings between dual polar spaces of type $D Q(2 n, \mathbb{K})$. Ann. Comb., to appear.

[18] B. De Bruyn. The hyperplanes of $D W(2 n-1, q)$ arising from embedding. preprint.

[19] B. De Bruyn. The hyperplanes of $D Q^{-}(7, \mathbb{K})$ arising from embedding. preprint.

[20] B. De Bruyn and H. Pralle. The hyperplanes of $D H\left(5, q^{2}\right)$. Forum Math., to appear.

[21] W. M. Kantor. Ovoids and translation planes. Canad. J. Math. 34 (1982), 1195-1207.

[22] C. M. O'Keefe and T. Penttila. Ovoids of PG(3,16) are elliptic quadrics. J. Geom. 38 (1990), 95-106.

[23] C. M. O'Keefe and T. Penttila. Ovoids of PG $(3,16)$ are elliptic quadrics, II. J. Geom. 44 (1992), 140-159. 
[24] G. Panella. Caratterizzazione delle quadriche di uno spazio (tridimensionale) lineare sopra un corpo finito. Boll. Un. Mat. Ital. 10 (1955), $507-513$.

[25] A. Pasini. Embeddings and expansions. Bull. Belg. Math. Soc. Simon Stevin 10 (2003), 585-626.

[26] S. E. Payne and J. A. Thas. Finite Generalized Quadrangles. Research Notes in Mathematics 110, Pitman, 1984.

[27] T. Penttila and B. Williams. Ovoids of parabolic spaces. Geom. Dedicata 82 (2000), 1-19.

[28] H. Pralle. Hyperplanes of dual polar spaces of rank 3 with no subquadrangular quad. Adv. Geom. 2 (2002), 107-122.

[29] E. E. Shult. Generalized hexagons as geometric hyperplanes of near hexagons. In "Groups, Combinatorics and Geometry" (eds. M. Liebeck and J. Saxl), Cambridge Univ. Press (1992), 229-239.

[30] E. E. Shult. On Veldkamp lines. Bull. Belg. Math. Soc. Simon Stevin 4 (1997), 299-316.

[31] E. E. Shult and A. Yanushka. Near $n$-gons and line systems. Geom. Dedicata 9 (1980), 1-72.

[32] J. A. Thas and S. E. Payne. Spreads and ovoids in finite generalized quadrangles. Geom. Dedicata 52 (1994), 227-253.

[33] J. Tits. Ovoïdes et groupes de Suzuki. Arch. Math. 13 (1962), 187-198.

[34] H. Van Maldeghem. Generalized Polygons. Monographs in Mathematics 93. Birkhäuser, Basel, Boston, Berlin, 1998. 